International Journal of Engineering \& Technology, 9(1)(2020) 202-210
International Journal of Engineering \& Technology
SPC
Website: www.sciencepubco.com/index.php/IJET
Research paper

\title{
Voltage regulation and minimization of loses in the presence of renewable energy sources on the medium voltage network
}

\author{
M. Dib ${ }^{1}{ }^{*}$, A.Nejmi ${ }^{1}$, M.Ramzi $^{1}$, M. Elhafyani ${ }^{2}$ \\ ${ }^{1}$ Laboratory of Automatic, Energy Conversion and Microelectronics, Faculty of Science and Technology, University \\ Sulan Moulay, Slimane, Beni Mellal, Morocco \\ ${ }^{2}$ Laboratory of Electrical Engineering and Maintenance, University Mohammed 1st, High School of Technology, Oujda, Morocco \\ *Corresponding author E-mail: m.dib@onee.ma
}

\begin{abstract}
The Moroccan electricity network has been designed and sized to work with high power outputs connected to the transmission network. This it delivers a unidirectional power flow from production to consumption. In this configuration, the electricity network carries electricity from production plants mainly connected to the transmission network to customers through the distribution network. Therefore, voltagesetting devices on the distribution network such as load-adjustable power transformers and medium voltage MV capacitor banks are set and operated. The inclusion of the Decentralized Generation (DG) based on renewable energies can induce a reversal of the power flow on the distribution network. This raises problems of voltage regulation in the presence of renewable energy sources. This article shows the limit of the current voltage regulation deployed solutions and exposes a solution for adjusting the voltage on the distribution grid in the presence of renewable energy sources connected to medium voltage MV. We will apply ETAP software to a real network located in the eastern zone for the conducted investigation. Later, an optimization approach aiming to minimize losses and improve the voltage plan will be presented at the end.
\end{abstract}

Keywords: Renewable Energy Sources; Decentralized Generation; Adjustment of the Voltage; Loss Optimization; Medium Voltage.

\section{Introduction}

Electrical power transmission and distribution systems operate at multiple voltage levels. Those voltage levels constitute an important control parameter. The distribution network operators are responsible for regulating the voltage delivered to the consumer within legal limits. HV / MV (OLTC) power transformer are used between these voltage levels to regulate and maintain the tension.

Since the transformation ratio of the MV/LV distribution transformers is not continuously adjustable, the choice of the appropriate voltage measurement depends on the installation location: the further it is from the substation, the lower the transformation ratio will be. Without decentralized injection, the voltage and the consumption decreases as one moved away from the LV/MV transformer station. The economic optimization of the network, as well as the respect of the operating conditions were then possible by decreasing the section of the conductors in end lines [1-2-3].

With renewable energy sources, variable injections of significant power can be connected to these sections. Thus, the voltage profile can be reversed and the section of the conductors proves to be insufficient. Depending on the power flows of consumers and producers, the currents can decrease, reverse or even increase in the opposite direction. The balance sheet of online losses becomes a complex task both analytically and for the billing phase [4-5].

Adjusting the voltage at the substation by dynamically correcting the voltage regulators' set points cannot always be sufficient to reduce the voltage to any point in the network. Acting on the reactive power is then an additional mean of local correction of the voltage, which then influences the balance of losses.

The Integration of decentralized generation can reverse the direction of power flow. Thus, sending power to the transmission system. A strong penetration of this resource on a weak network can generate overvoltage at the end of distribution line. Thus, a revision of the voltage regulation of the distribution network is required. DGs are distinguished from centralized production units by the fact that they are most often connected to the distribution network, and by their "small size", since their main characteristic is intermittency [5-6].

The increase number in decentralized generation, which is highly variable and geographically dispersed, requires a new grid voltage plan that will allow conversion to the energy mix. Indeed, the role of the distribution network is to provide electrical power to customers connected in MV or LV by ensuring continuity of service and quality of the electric wave, in the best conditions of safety and at the best cost. Consequently, the distribution network manager operates at the design (structure, number, sizing ...) and operating rules [7-8] levels. 
This article shows the limit of the current regulation and exposes a vision of adjustment of the voltage on the distribution electricity grid in the presence of decentralized generations.

\section{Current regulation of voltage in distribution networks}

The principle of voltage regulation on a distribution network without decentralized generation is presented in Figure 1.

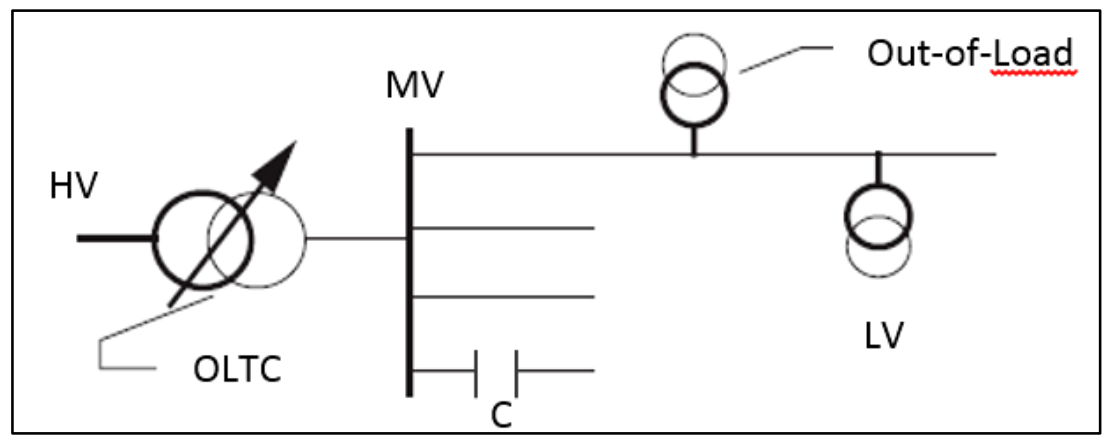

Fig. 1: Principle of Voltage Regulation on a Distribution Network without Productors.

The HV/MV on-load tap changer is the most commonly used voltage control in the MV distribution network. This device allows to adjust the voltage of the MV busbar setting a position on different taps along with the evolution of the loads and the fluctuations of the upstream voltage by adding or removing spirals [9-10].

The capacitor batteries installed at the source station allow injecting the reactive power into the busbar. This injection is capable of compensating the reactive power transferred via the transformer. Thus, the reactive power transfer from the upstream network is reduced. The capacitor bank compensation improves the power factor with respect to the transport network and consequently reduces voltage drops. This also minimizes the active losses of the transmission network since there are fewer current transits [10-11-12].

In Low Voltage (LV), the voltage setting is to use the out-of-load taps of the MV / LV transformers. The MV/LV transformer taps are used to compensate for all or part of the voltage drops on the MV networks [14].

The conventional voltage setting with the On-load tap changer OLTC does not adapt perfectly in the presence of DG. If a source station is connected with several feeds with DG non homogeneous insertion. The regulator in charge cannot always work properly to lift tension constraints. The voltage must not be very high to prevent the voltage on the feeders with DG exceeding the upper limit, or too low for the voltage on the feeders without DG to be higher than the lower limit. [14-15].

\section{Analysis of voltage drops and line losses}

The management of decentralized productions (DG) is an essential element to meet the needs of the good functioning of an active distribution network. The objective of this part is to make an approach of the optimal regulation of the voltage in the presence of the DGs within active distribution networks. This approach consists of minimizing active line losses in the MV network.

\subsection{Voltage drops}

To ensure, at any point in the network (MV or LV), a voltage satisfying the contractual and regulatory values, the manager is required to establish a voltage plan. This plan consists in using in MV the possibilities of setting the target voltage at the bus-bar of the HV/MV transformer supplying the network concerned and in LV the off-load setting of the MV/LV transformers. It is based on the general voltage profile of the MV feeders connected to the same HV/MV transformer. The connection of a production facility on an MV network induces, as a result of the active and reactive power injection, a modification of the energy that transits on the network [16]. The voltage profile calculation on a distribution network element is generally performed using the formula:

$\frac{\Delta \mathrm{U}}{\mathrm{U}_{\mathrm{n}}}=\frac{\mathrm{R} \cdot \Delta \mathrm{P}+\mathrm{X} \cdot \Delta \mathrm{Q}}{\mathrm{U}^{2} \mathrm{n}}$

(1)

$\Delta \mathrm{U} \%=\frac{\Delta \mathrm{U}}{\mathrm{U}_{\mathrm{n}}} \cdot 100=\frac{100}{\mathrm{U}^{2} \mathrm{n}} \cdot\left(\sum_{\mathrm{i}=1}^{\mathrm{m}} \Delta \mathrm{P}_{\mathrm{i}} \cdot \mathrm{R}_{\mathrm{i}}+\sum_{\mathrm{i}=1}^{\mathrm{m}} \Delta \mathrm{Q}_{\mathrm{i}} \cdot \mathrm{X}_{\mathrm{i}}\right)$

Where:

- $\quad \Delta \mathrm{U} / \mathrm{U}$ : relative voltage drop at the terminals of a network element.

- $\quad U_{n}$ : Nominal compound voltage.

- $\mathrm{R}$ and $\mathrm{X}$ : resistance and reactance of this network element.

- $\Delta \mathrm{P}$ : Active power transiting in the network element.

- $\quad \Delta \mathrm{R}$ : Reactive power passing through the network element.

\subsection{Electrical losses in an MV line}

The transport of electrical energy generates losses, due to the physical phenomenon of heating generated by conducting materials traversed by a current. "Joule" losses are proportional to the square of the current. Loses are higher as the transported current is important. 
Reducing losses in distribution and transmission lines is an important factor for the economic and technical assessment of the national electricity grid.

The formula expressing the losses on a departure is given by [17-18]:

$\Delta \mathrm{W}_{\mathrm{j}}=\int \Delta \mathrm{P}_{\mathrm{j}} \cdot \mathrm{dt}$

(3)

With:

$\Delta \mathrm{W}_{\mathrm{j}}=\frac{\mathrm{R}_{\mathrm{L}} \cdot \mathrm{L}}{\mathrm{N} \cdot \mathrm{U}^{2}} \times \int_{0}^{\mathrm{T}} \mathrm{S}^{2} \cdot \mathrm{dt}$

(4)

Where $\mathrm{R}_{\mathrm{L}}$ a linear resistance, $\mathrm{L}$ the line length, I the current flowing through the line, $\mathrm{U}$ the rated voltage and $\mathrm{S}$ the apparent power carried by the line.

\subsection{Optimum regulation of the voltage}

The objective function is given by [14-16]:

$\min _{\Delta \mathrm{X}}\left\{\alpha \cdot \mathrm{P}_{\mathrm{j}}+\beta \cdot \mathrm{S}_{\mathrm{v}}\right\}$

(5)

With $\mathrm{S}_{\mathrm{v}}=\sum_{\mathrm{i}}^{\mathrm{Nbus}}\left|\mathrm{V}_{\mathrm{i}}-\mathrm{V}_{\mathrm{N}}\right| \mid$ is the sum of the differences between the voltages measured at the bus and the rated voltage. By putting as constraint:

$\mathrm{V}_{\mathrm{i}, \min }<\mathrm{V}_{\mathrm{i}}<\mathrm{V}_{\mathrm{i}, \max }$

Relationships between the $\mathrm{X}$ vector and the parameters to be minimized are presented as follows [20-21]:

$\left|\Delta \mathrm{V}_{\mathrm{i}}\right|=\mathrm{K}_{\mathrm{iP}} \cdot \Delta \mathrm{P}_{\mathrm{DER}}+\mathrm{K}_{\mathrm{iQ}} \cdot \Delta \mathrm{Q}_{\mathrm{DER}}+\mathrm{K}_{\mathrm{in}} \cdot \Delta \mathrm{n}_{\mathrm{OLTC}}$

(6)

$\left|\Delta \mathrm{P}_{\mathrm{j}}\right|=\mathrm{L}_{\mathrm{PjP}} \cdot \Delta \mathrm{P}_{\mathrm{DER}}+\mathrm{K}_{\mathrm{PjQ}} \cdot \Delta \mathrm{Q}_{\mathrm{DER}}+\mathrm{K}_{\mathrm{Pjn}} \cdot \Delta \mathrm{n}_{\mathrm{OLTC}}$

(7)

Where :

- $\mathrm{K}_{\mathrm{ik}}, \mathrm{L}_{\mathrm{Pjk}}:$ Sensitivity coefficients.

- $\mathrm{n}_{\text {OLTC }}$ : Number of taps.

\section{Experimental study}

In what follows, the study will focus on a real network (Oujda source substation) that contains three $22 \mathrm{kv}$ feeders, one of which is connected directly to a 2 MW BioGas decentralized generation. The objective is to visualize the voltages at different nodes of the departures (the buses) and to compare and analyse the plan when connecting other decentralized units. This one line diagram is drawn using the ETAP software, which allows the study and simulation of electrical networks [14].

Figure 2 illustrates the diagram of the studied network: 


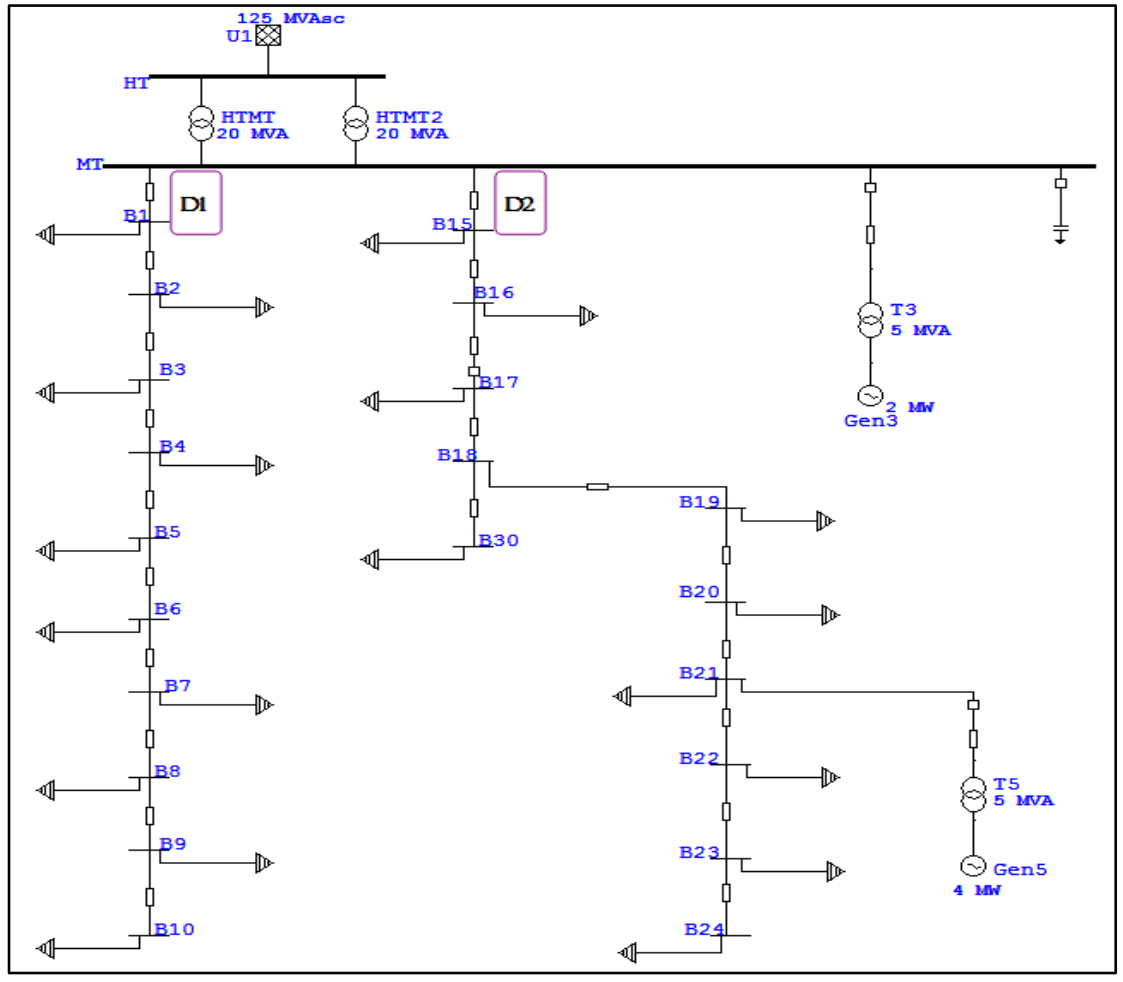

Fig. 2: Example of an Image with Acceptable Resolution.

\subsection{Network without DG connected}

The first scenario is to visualize the power flow on our study network by the ETAP software, to record the voltages and the losses data. For this case, only the on-load tension control of $60 / 22 \mathrm{kV}$ transformers has been activated without compounding. The simulation showed that the tap adjuster operated 3 times to maintain the voltage at the $22 \mathrm{kv}$ busbar in the dead band $[-0,4 \% ; 0,4 \%]$ autour de $1.0 \mathrm{pu}$; Yet the voltage drop persists at the end of the lines.

\subsection{Network with one DG connected to busbar}

The second scenario consists in connecting the Biogas production to the busbar for a power of $2 \mathrm{MW}$ with $\cos \phi=1$. The simulation showed that the load adjuster operated once to maintain the tension in the belt due to the DG's injection of power. This tap change will decrease the voltage level for the distant points.

\subsection{Network with two DGs and No $Q$ compensation}

The third scenario is to connect a DG operating with $3 \mathrm{MW}$ to bus 21 in feeder D2 with $\cos \phi=1$. The simulations showed an improvement of the voltage level on the buses of departure D2 and especially in the vicinity of the connection point of the DG, and no improvement on the departure without DG. The OLTC did not react.

\subsection{Network with two DGs and $Q$ compensation}

The fourth scenario is to change the production mode for the DG initially connected to D2 by activating the reactive energy compensation (Swing). In this case the DG operated with an active power of $2.8 \mathrm{MW}$ and reactive of $0.5 \mathrm{Mvar}$.

\section{Simulation results}

\subsection{Signal quality}

Table 1 shows the variations of the voltage in relation to the nominal value $22 \mathrm{kv}$ at the pilot nodes. In our case, the pilot nodes are the DG busbar, the end of the line of the feeder D1 and the connection point at D2.

Table 1: Tension Values in Pilot Nodes

\begin{tabular}{llll}
\hline Voltage evolution in pilot nodes (\%) & $1^{\text {st }}$ Case & $2^{\text {nd }}$ Case & $3^{\text {rd }}$ Case \\
\hline Busbar JB & 100.37 & 99.91 & 99.93 \\
B10-end of D1 & 94.86 & 94.42 & 100.05 \\
B21-DG-D2 & 96.82 & 96.37 & 94.44 \\
B24-end of D2 & 96.47 & 96.02 & 98.39 \\
\hline
\end{tabular}

Figure 3 shows the voltage level along the D1 and D2 feeders. 


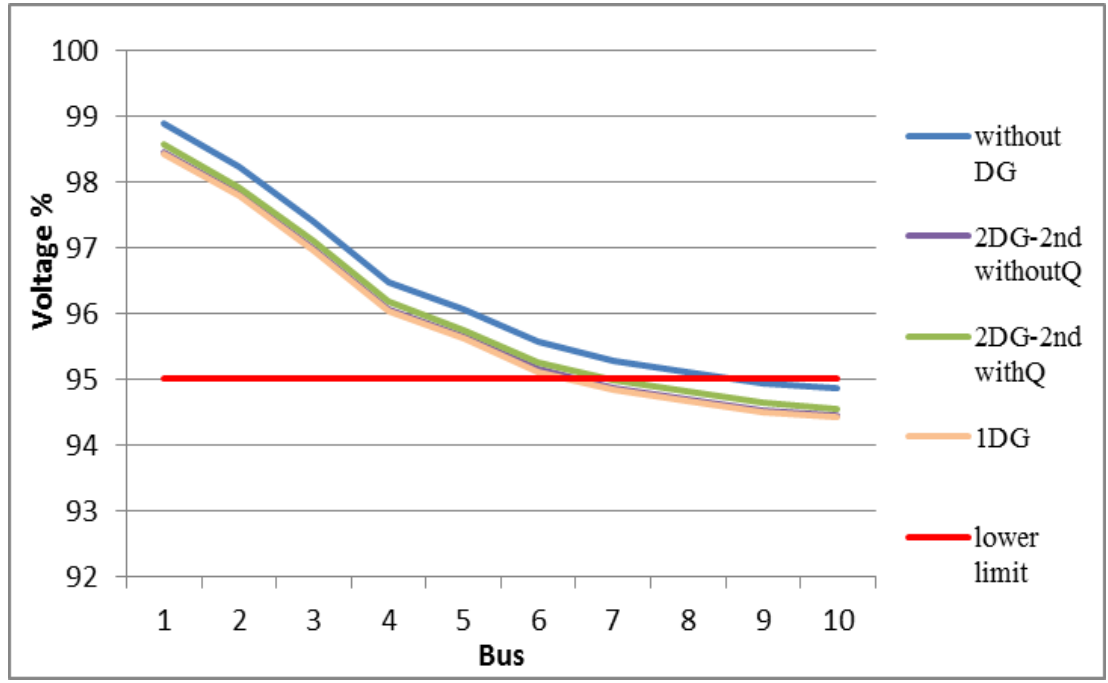

Fig. 3: Voltage Evolution along the D1 Feeder.

This figure reveals a voltage violation on the D1 start that has no injection. The voltage along the D1 start improves when the DGs connected at the D2 start injects active and reactive powers. This improvement remains insufficient compared to the lower limit. Thus, the reactive power injection can increase the joules losses on the D1 start.

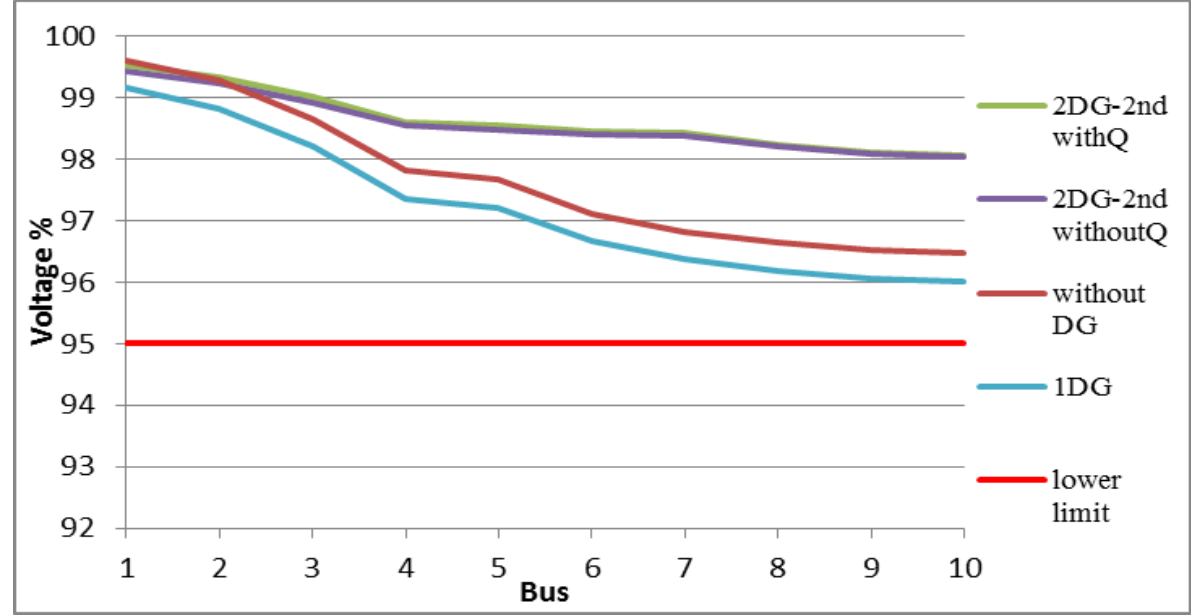

Fig. 4: Voltage Evolution along the D2 Feeder.

Voltage improvement along the departure D2 is depicted in figure 4. The voltage profile depends on the injection of the active and reactive powers of the decentralized productions connected to this output. Blocking this injection, can increase the joules losses on this departure D2.

\subsection{Looses}

The following table and figure show the losses for each scenario.

Table 1: Real Losses

\begin{tabular}{llll}
\hline Losses in Kw & $1^{\text {st }}$ Case & $2^{\text {nd }}$ Case & $3^{\text {rd }}$ Case \\
\hline HV/MV & 70.95 & 59.26 & 44.78 \\
Transformers losses & 983.74 & 1003.66 & 45.49 \\
Total of losses & & 887.05 & 883.11 \\
\hline
\end{tabular}




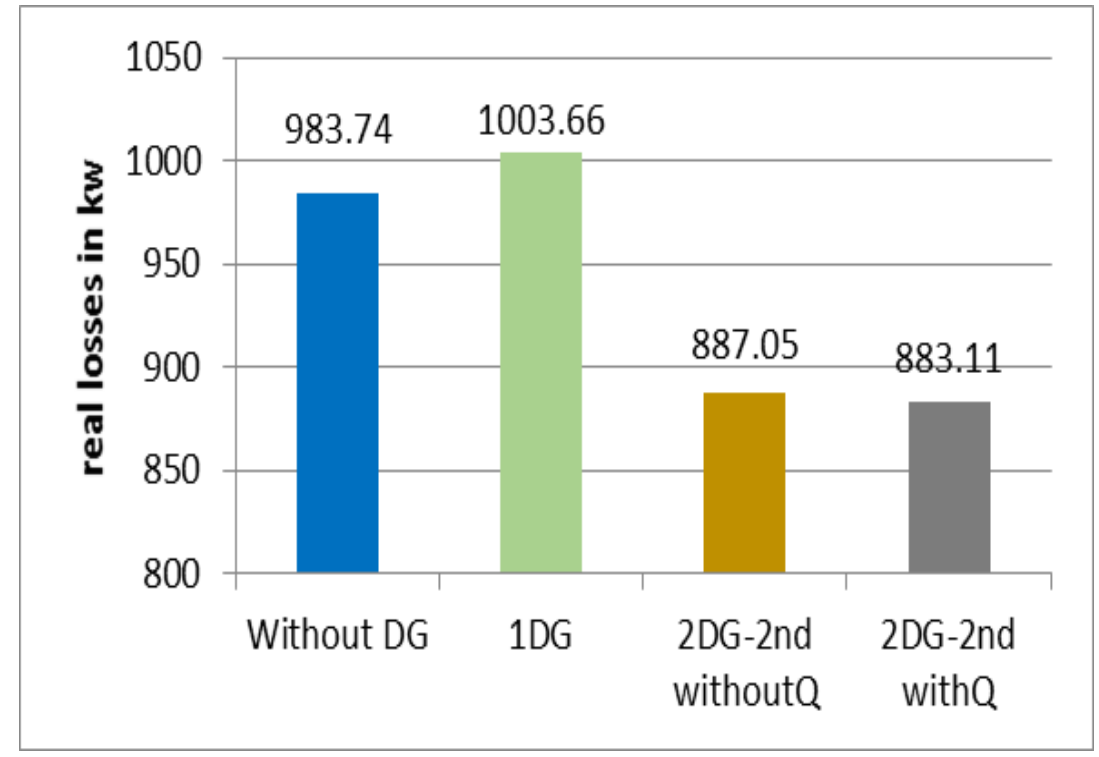

Fig. 5: Total of Real Losses in Network.

The results obtained show that the limit of the current voltage regulation in the presence of the DGs and the limit of the on-load tap changer in the presence of DGs on a departure has no action to improve the voltage level of the departure without DG.The connection of the decentralized production units improves the voltage level on the nodes of the $22 \mathrm{kv}$ feeders. The coordination between the OLTC and the active and reactive powers will improve the voltage plan and minimize losses. Not to mention that the connection of DGs must be next to the important loads to avoid the circulation of the flow generated in several lines and over long distances. The size and location of the DGs influences the voltage level and the losses. For this reason, we will propose in the following section an approach for the optimal regulation of the voltage by the participation of the OLTC, the active and reactive powers of the DGs.

\subsection{Voltage regulation}

The optimization method is carried out for 3 cases:

- First case: $\alpha=1, \beta=0$

- Second case: $\alpha=1, \beta=1$

- Third case: $\alpha=20, \beta=1$

The non-null value of $\alpha$ allows the minimization of losses. Favoring one objective is done by increasing its weighting over others. The constraints will be affected on the MV 22kv busbar and on two pilot nodes number 10 and 21 . The first node corresponds to the connection point of the DG and the other node at the end of line D1. The control of the tension at these 3 points leads directly to a healthy plan of tension.

The band values introduced on ETAP are:

- $100 \%<\mathrm{V}_{\mathrm{MV}}<102 \%$

- $\quad 95 \%<\mathrm{V}_{10}<105 \%$

- $\quad 98 \%<\mathrm{V}_{21 \mathrm{DG}}<102 \%$

Optimal Flow simulation is launched with the objective of minimizing losses across the entire network, and the following results have been found.

It is noted that the constraints on the tension are well respected. For line losses, there is a clear improvement over the situation before optimization for cases 1 and 3. Below the three tables 3, 4 and 5showing the results: 


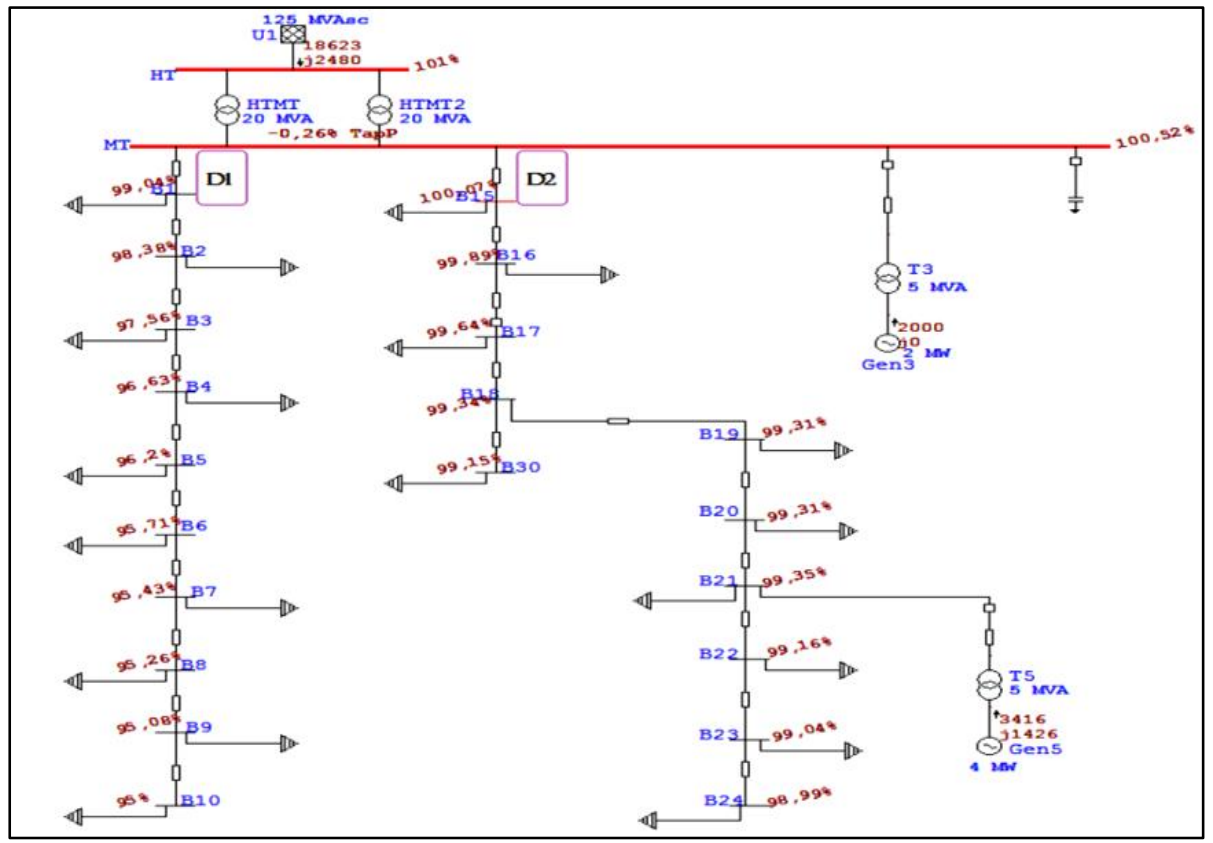

Fig. 6: Optimal Flow Analysis for Case 1.

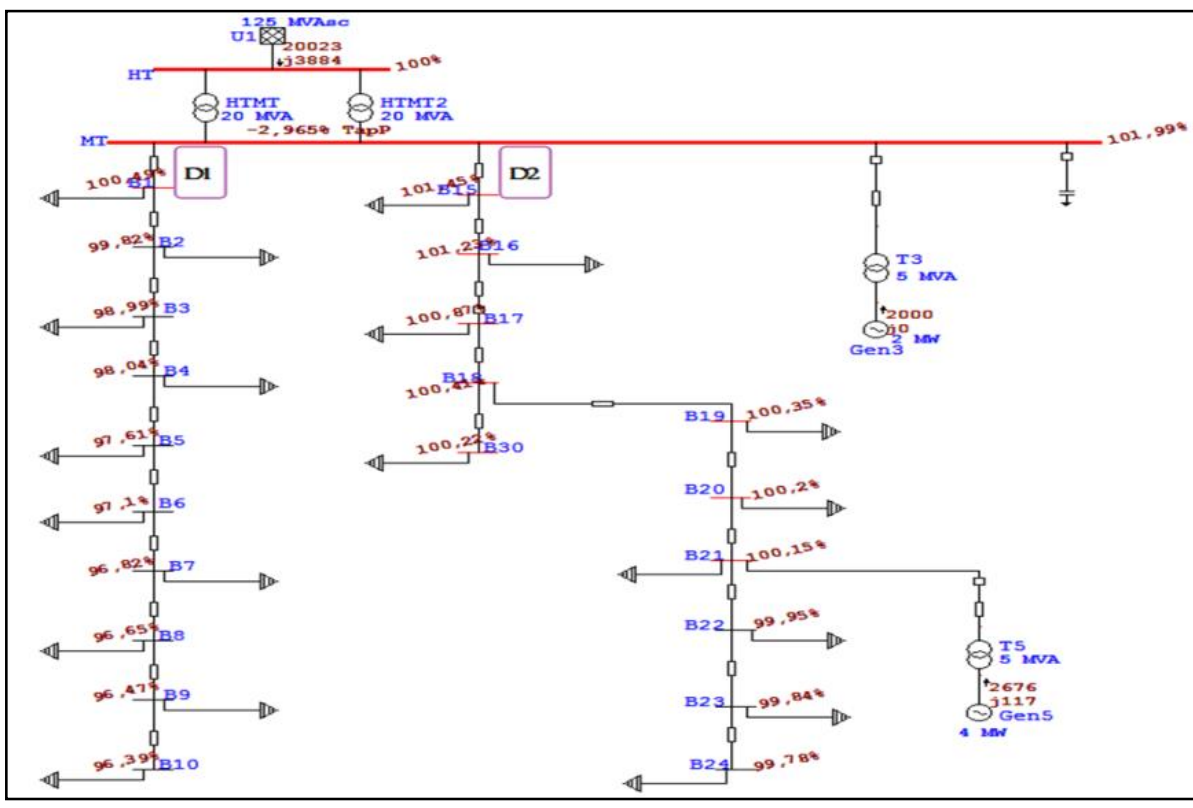

Fig. 7: Optimal Flow Analysis for Case 2.

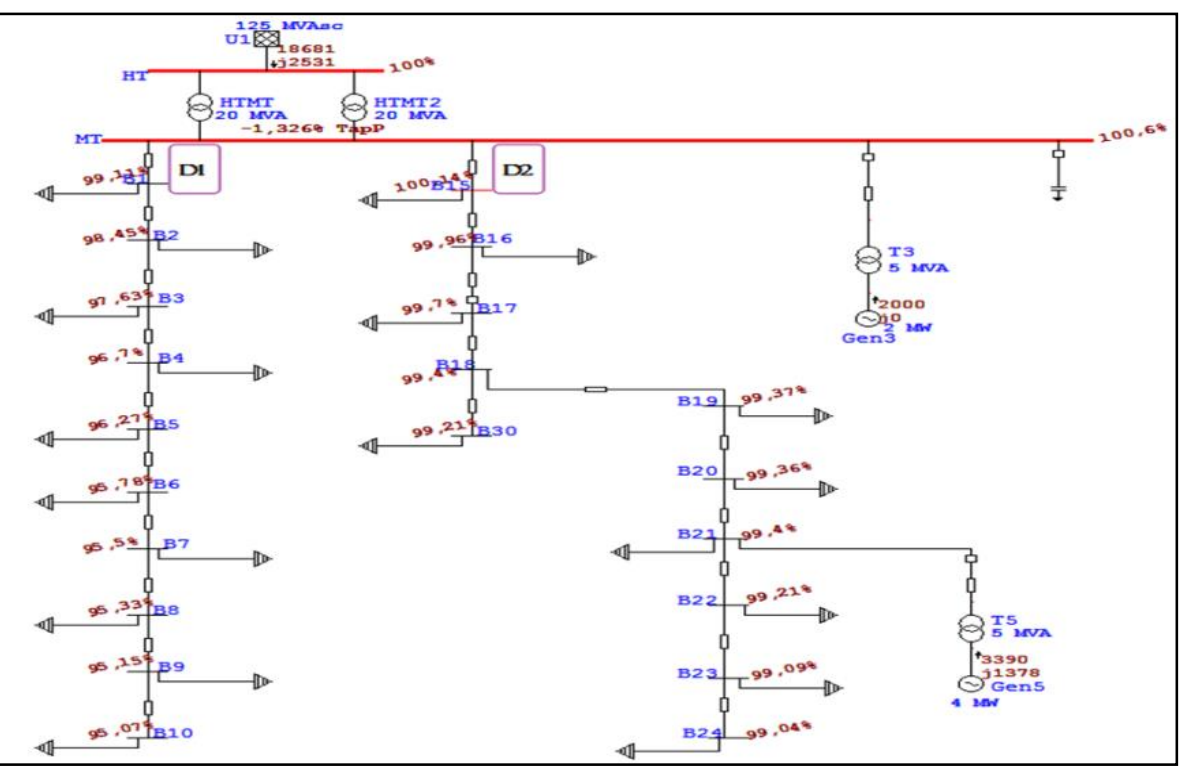

Fig. 8: Optimal Flow Analysis for Case3. 
Table 3: Result of Line Losses in Kw

\begin{tabular}{lllll}
\hline without DG & 2DG connected & & & \\
\hline Real Losses & before optimisation & after optimisation case 1 & after optimisation case 2 & after optimisation case 3 \\
938.74 & 883.11 & 878 & 925 & 880 \\
\hline
\end{tabular}

For joules losses, there is a clear improvement over the situation before optimization for cases 1 and 3 . This is explained by the fact that the values of the alpha factor are greater than or equal to those of the Beta factor. The losses are high in case 2 compared to the state without optimization. This is explained by the fact that the control of the circulation of the reactive powers (alpha $=0$ ) was inhibited and the voltage level was controlled only.

The optimal powers generated by the second DG connected to the D2 start are recorded in table 4:

Table 4: Positions OLTC

\begin{tabular}{|c|c|c|c|}
\hline without DG & 2DG connected & & \\
\hline $\begin{array}{l}\text { OLTC Position } \\
-3\end{array}$ & $\begin{array}{l}\text { before optimisation } \\
-2\end{array}$ & $\begin{array}{l}\text { after optimisation case } 1 \\
-1\end{array}$ & $\begin{array}{l}\text { after optimisation case } 2 \\
-6\end{array}$ \\
\hline
\end{tabular}

The load adjuster participated in the voltage regulation depending on the case. In case 2, it worked six times to maintain the $22 \mathrm{kv}$ JDB voltage in the set band.

Table 5: Losses Leads to the Generation of Reactive Power from the 2nd DG

\begin{tabular}{|c|c|c|c|c|c|c|c|}
\hline \multicolumn{8}{|c|}{ DG connected to D2 } \\
\hline \multicolumn{2}{|c|}{ before optimisation } & \multicolumn{2}{|c|}{ after optimisation case 1} & \multicolumn{2}{|c|}{ after optimisation case 2} & \multicolumn{2}{|c|}{ after optimisation case 3} \\
\hline $\mathrm{P}$ en $\mathrm{Mw}$ & Q en Mvar & $\mathrm{P}$ en $\mathrm{Mw}$ & Q en Mvar & $\mathrm{P}$ en Mw & Q en Mvar & $\mathrm{P}$ en Mw & Q en Mvar \\
\hline 2.8 & 0.5 & 3.42 & 1.43 & 2.77 & 0.12 & 3.39 & 1.38 \\
\hline
\end{tabular}

Favoring the minimization of losses leads to the generation of reactive power from the second DG. The Q injected into the network relieves the transformers of the HV/MV substation by participating in the improvement of the voltage on the busbar and the nodes of the other feeder, given the number of plugs changed by the OLTC.

Losses can be further minimized by finding the optimal location of the DG and by planning the development of the $22 \mathrm{kv}$ network.

\section{Conclusion}

In this article, we have presented the Moroccan electrical system and the voltage regulation mode at the distribution network. This setting mode has shown its limit in the presence of decentralized generations.

The connection of these Generations DG based on renewable energy sources, can change the operation of the distribution network. This change affects the conventional voltage-setting mode, and will therefore require a new voltage regulation mode through the participation of the decentralized productions.

The proposed approach based on a coupling between the current network control means and DG facilities. It involves using existing tuning tool son the distribution networks and adjusting the active and reactive power of renewable energy sources to resolve voltage violations and minimize joules losses.

This participation will also increase the insertion rate of these sources in the distribution network, particularly in MV medium voltage. The minimization of these losses depends on the structure of the network, the location and the size of the decentralized productions. However, a lot of work and simulations have yet to be done to evaluate the feasibility of this approach.

Among these works is to study the optimization of the losses according to the structure of the network, the location and the size of the decentralized productions and by introducing other devices of adjustment like the regulators of tension installed on the MV lines.

\section{References}

[1] M. Brenna, E. Berardinis, F. Foiadelli, G. Sapienza and D. Zaninelli (2010), "Voltage Control in Smart Grids: An Approach Based on Sensitivity Theory," Journal of Electromagnetic Analysis and Applications,Vol. 2 No. 8, 2010, pp. 467-474. https://doi.org/10.4236/jemaa.2010.28062.

[2] Caldon, R.; Coppo, M.; Turri, R. (2013), "Coordinated voltage control in MV and LV distribution networks with inverter-interfaced users," PowerTech (POWERTECH), 2013 IEEE Grenoble, vol., no., pp.1,5, 16-20 June 2013. https://doi.org/10.1109/PTC.2013.6652491.

[3] Capitanescu, F.; Bilibin, I.; Romero Ramos, E. (2014), "A Comprehensive Centralized Approach for Voltage Constraints Management in Active Distribution Grid," Power Systems, IEEE Transactions on, vol.29, no.2, pp.933,942, March 2014. https://doi.org/10.1109/TPWRS.2013.2287897.

[4] Christakou, K.; Tomozei, D.-C.; Le Boudec, J.-Y.; Paolone, M. (2014), "GECN: Primary Voltage Control for Active Distribution Networks via RealTime Demand-Response,"Smart Grid, IEEE Transactions on , vol.5,no.2, pp.622,631, March 2014. https://doi.org/10.1109/TSG.2013.2275004.

[5] Voltage Control Techniques of Distribution Networks Using On-Load Tap Changer Transformers", Journal of Electric Power Science and Technology, China, 2011.

[6] Advanced Voltage Control Strategy for On-Load Tap-Changer Transformers with Distributed Generations", 46th International Universities Power Engineering Conference, Soest, Germany, UPEC 2011.

[7] Olivier R, Andreea V, Yvon B (2006). Coordinated voltage control in distribution networks using distributed generation. Transmission and Distribution Conference IEEE.

[8] Milos M, Dejan M (2014). A Practical Case of Determining the Maximum allowed PV Plant Connection Power Using Different Reactive Power Control Concepts. IEEE.

[9] Sudarshan D (2015). Renewable energy development in Australia: Regulatory to technical changes. Asia-Pacific power and energy engineering conference; IEEE.

[10] Christakou K, Dan-Cristian T, Jean-Yves, L.B(2014). Primary Voltage Control for Active Distribution Networks via Real-Time Demand-Response. IEEE. https://doi.org/10.1109/TSG.2013.2275004.

[11] Chao, G (2011). Voltage Control Techniques of Distribution Networks Using On-Load Tap Changer Transformers. Journal of Electric Power Science and Technology. China.

[12] Peter F, Ignacio T, Jutta H (2015). Optimized Regulation of Dispersed Generation Units for Minimization of Reactive Power Consumption. IEEE.

[13] Frano T, Kristina B, Marko D (2014). Reactive Power Optimization Based on Load Profile Partitioning. ENERGYCON 2014; Croatia. 
[14] Konstantinos, K (2017). Decentralized Voltage Regulation in radial Medium Voltage Networks with high presence of Distributed Generation. Porto Journal of Engineering.

[15] Pedro C, Pedro C, Luis F (2008). Distributed reactive power generation control for voltage rise mitigation in distribution networks. IEEE.

[16] Abla G. Impact of the Expansion of the Production of Solar Power on the Low Voltage Network in Egypt.

[17] Jean-Luc F, Laurent K (2010). Raccordement de la production décentralisée aux réseaux de distribution-aspects techniques. Techniques de l'ingénieur.

[18] Armend Y, Lutfij D, Arta Q (2014). Impacts of distributed generation in energy losses and voltage drop in 10 Kvline in the distribution system. ENERGYCON.

[19] Rexhep S, Dimitar B, Erdet N (2016). Model for estimation of the effects of new generators connected to existing distribution network of Kosovo. IEEE.

[20] Alberto B, Mauro B, Samuele G, Stefano M, Carl A, Mario, P.F.S (2010). Short-term scheduling and control of active distribution systems with high penetration of renewable resources. IEEE systems journal.

[21] R.Muthu K (2016). Optimal Power Flow of Distribution Network. International Journal of Computer \& Mathematical Sciences. 